\title{
Influence of T1-Weighted Signal Intensity on FSL Voxel-Based Morphometry and FreeSurfer Cortical Thickness
}

\author{
(D) S. Chung, (D)X. Wang, and (D)Y.W. Lui
}

\begin{abstract}
SUMMARY: The effect of T1 signal on FSL voxel-based morphometry modulated GM density and FreeSurfer cortical thickness is explored. The techniques rely on different analyses, but both are commonly used to detect spatial changes in GM. Standard pipelines show FSL voxel-based morphometry is sensitive to $\mathrm{Tl}$ signal alterations within a physiologic range, and results can appear discordant between FSL voxel-based morphometry and FreeSurfer cortical thickness. Care should be taken in extrapolating results to the effect on brain volume.
\end{abstract}

ABBREVIATIONS: $\mathrm{CT}$ = cortical thickness; $\mathrm{mGM}=$ modulated $\mathrm{GM}$ density; $\mathrm{mTBI}=$ mild traumatic brain injury; $\mathrm{VBM}=$ voxel-based morphometry

C ortical segmentation methodologies vary and are used throughout neuroimaging research as well as, increasingly, in clinical care. Two commonly used methods to study cortical GM are FMRIB Software Library voxel-based morphometry (FSL-VBM [http://fsl.fmrib.ox.ac.uk/fsl/fsl-4.1.9/fslvbm/index. html] $)^{1}$ and FreeSurfer (http://surfer.nmr.mgh.harvard.edu) cortical thickness (CT). ${ }^{2}$ FSL-VBM outputs a 3D map of modulated GM density (mGM), and FreeSurfer-CT analysis outputs 1D measurements around the cortical ribbon. Both are commonly interpreted as informing cortical volume, though there are instances where volume was increased based on one technique and decreased based on the other, ${ }^{3,4}$ suggesting that factors beyond brain volume contribute to results.

GM and WM contrast naturally contributes to successful segmentation; however, the extent to which changes in cortical T1 signal affect $\mathrm{mGM}$ and CT is not known. Understanding this relationship is critical for an appropriate interpretation. This study explored the effect of subtle T1 signal alterations within a physiologic range on FSL-VBM ${ }^{1}$ and FreeSurfer- $\mathrm{CT}^{2}$ by using standard

Received June 24, 2016; accepted after revision October 26.

From the Center for Advanced Imaging Innovation and Research (CAI2R), Department of Radiology, and Bernard and Irene Schwartz Center for Biomedical Imaging, Department of Radiology, New York University School of Medicine, New York, New York.

This work was supported by grant R01 NS039135-11 from the National Institute for Neurological Disorders and Stroke, a component of the National Institutes of Health. This work was also performed under the rubric of the Center for Advanced Imaging Innovation and Research (CAI2R, www.cai2r.net), a National Institute of Biomedical Imaging and Bioengineering Biomedical Technology Resource Center (NIH P41 EB017183).

Please address correspondence to Yvonne W. Lui, MD, 660 1st Ave, Room 745, New York, NY 10016; e-mail: yvonne.lui@nyumc.org

- Indicates open access to non-subscribers at www.ajnr.org

http://dx.doi.org/10.3174/ajnr.A5053 processing pipelines. We also illustrate discordance between the techniques through individual clinical examples.

\section{MATERIALS AND METHODS}

This study is in compliance with our Department of Radiology institutional review board. MPRAGE was performed at 3T (Skyra [Siemens, Erlangen, Germany]; FOV, $256 \times 256 \mathrm{~mm}^{2}$; resolution, $1 \times 1 \times 1 \mathrm{~mm}^{3}$; matrix, $256 \times 256$; sections, 192; TR, $2100 \mathrm{~ms}$; TE, 3.19 ms; TI, $900 \mathrm{~ms}$; bandwidth, $260 \mathrm{~Hz}$ /pixel; flip angle, $8^{\circ}$ ).

\section{Signal Intensity Simulation}

MPRAGE images from a healthy 25-year-old man were used to generate simulated signal changes within the frontal operculum (Fig 1). Forty percent of voxels within the ROI were randomly selected for signal intensity alteration of up to $\pm 20 \%$ ( $5 \%$ increments), covering a physiologic T1 range of GM. Pearson correlation coefficient was measured (5\% significance level).

\section{Clinical Examples}

Several clinical cases were selected to illustrate concordant and discordant results: 1) acute and chronic infarcts; 2) healthy 19-year-old and 50-year-old patients; and, 3) a 28-year-old man with mild traumatic brain injury (mTBI) and an age- and sex-matched control patient.

\section{Image Analysis}

All images underwent denoising. ${ }^{5}$ Standard FSL-VBM (v1.1) processing steps included brain extraction, manual editing, automated tissue-type segmentation, nonlinear registration to GM template, modulation, and smoothing with isotropic Gaussian kernel $(\sigma, 3 \mathrm{~mm})$. FreeSurfer (v5.3.0) analysis was performed to estimate regional CT.

ROIs were drawn around areas of acute and chronic infarct 
in the native space and compared with contralateral normalappearing analogous brain. For the control group and patients with TBI, a precuneal ROI from the Harvard-Oxford atlas was interrogated because previous studies report age- ${ }^{6}$ and TBI-associated $^{7}$ morphometry changes in this region. ROIs drawn in the native space were warped to the target space by using transform matrices created by FSL-VBM and FreeSurfer-CT.

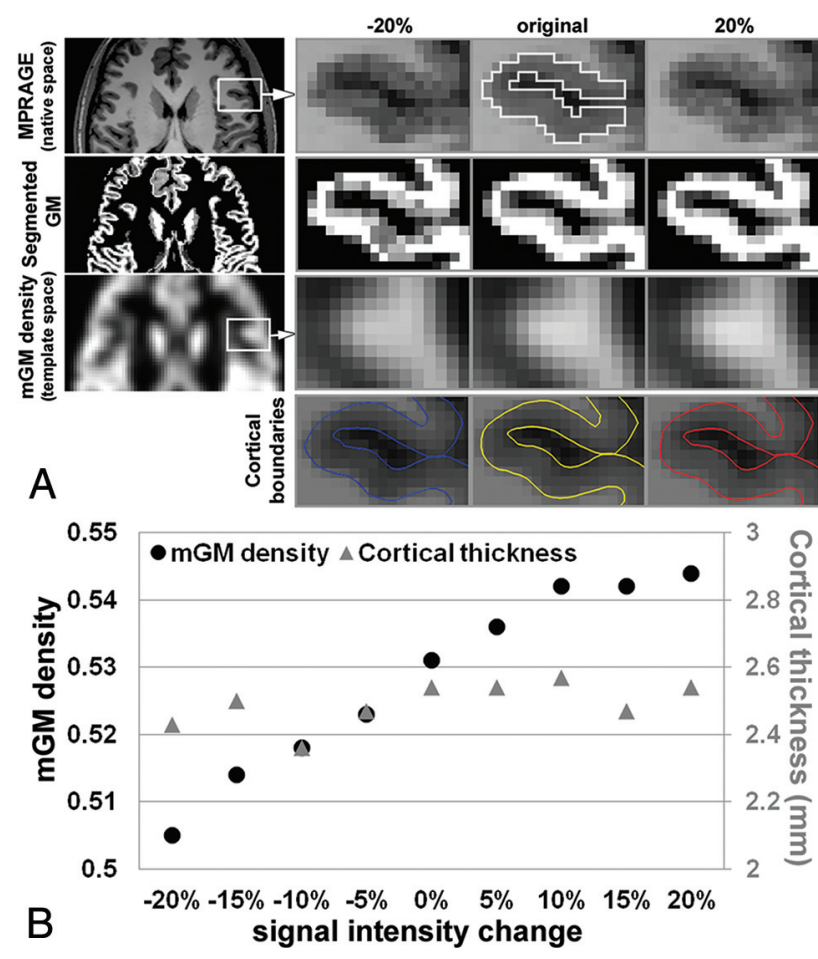

FIG 1. Simulated cortical signal change. A, First row (left to right), Representative MPRAGE in a healthy control patient (25-year-old man). Forty percent of voxels through 7 slices within the ROI were randomly selected and used to simulate changes in $\Pi$ signal intensity via decreasing and increasing voxel intensity to $20 \%$ in increments of $5 \%$. Selected ROI from the frontal operculum for simulating signal change is shown in the original image (white solid line). Second row, Corresponding segmented GM maps in the native space. Third row, mGM maps in the template space and, Fourth row, corresponding cortical boundaries from FreeSurfer. B, High correlation between signal intensity and $\mathrm{MGM}$ is observed (black $\operatorname{dots}[R=0.964 ; P<.001])$, but no relationship is seen between signal intensity and $\mathrm{CT}$ (gray triangles).

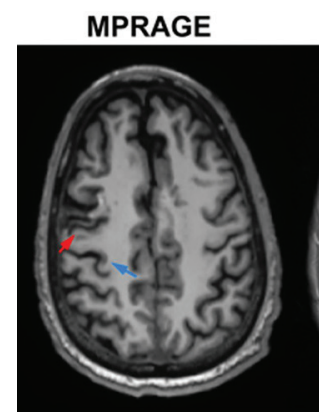

A
FLAIR

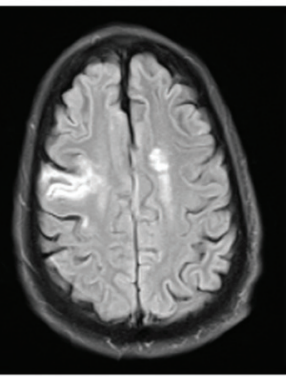

B
DWI/ADC

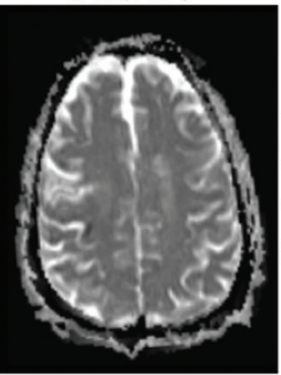

C

\section{RESULTS}

Simulation results show a strong correlation between signal intensity and $\mathrm{mGM}(R=0.964 ; P<.001)$. No correlation is present between signal intensity and CT (Fig $1 B)$.

Concordant and discordant results are illustrated in Figs 2 and 3. The acute infarct showed $23 \%$ higher $\mathrm{mGM}$ and $52 \%$ higher CT compared with the contralateral analogous brain. The older healthy patient showed 30\% lower mGM and $8 \%$ lower CT in the precuneus compared with the younger control patient. Discordant results included area of chronic infarct, demonstrating 26\% higher $\mathrm{mGM}$ and $43 \%$ lower CT compared with the contralateral normal-appearing analogous brain. In the patient with mTBI, mGM was $12 \%$ lower and CT was $2 \%$ higher in a precuneal ROI compared with a matched control patient.

\section{DISCUSSION}

FSL-VBM is sensitive to T1 signal variations within a clinically relevant range, which was not found to be true for FreeSurfer-CT. Although mGM and CT are completely different measures, both are commonly used to assess GM volume. Discordant results between FSL-VBM and FreeSurfer-CT analyses may result from T1 effects on mGM. This observation is of clinical and research importance because there are myriad conditions that affect $\mathrm{T} 1$ signal. Careful interpretation of FSL-VBM is warranted, particularly in the setting of discordant findings.

Several FSL-VBM methodologic steps are worth comment: 1) bias correction may alter T1 signal, and 2) tissue-type segmentation can affect output mGM. All images underwent identical bias correction before both analyses, and we demonstrated the effect of T1 signal change on mGM and CT by applying standard pipelines to recreate commonly used approaches that have widespread availability. Future optimization is warranted to achieve accurate detection of pathology.

Other methods of cortical segmentation are not specifically addressed here. Whether a similar dependence on T1 signal is present in VBM approaches such as SPM12 (http://www.fil.ion. ucl.ac.uk/spm/software/spm12) is not known. The cases provided as part of this report are not meant as generalizable results regarding the specific pathologies described, but instead serve as in vivo examples of the phenomenon of discordance in terms of interpreting FSL-

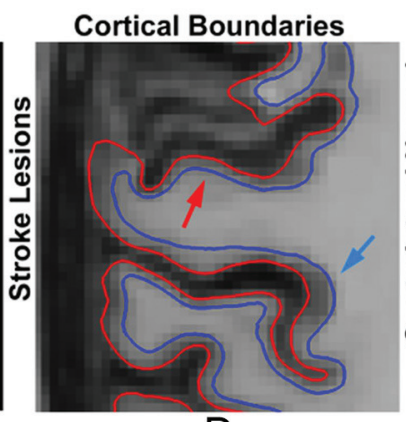

D

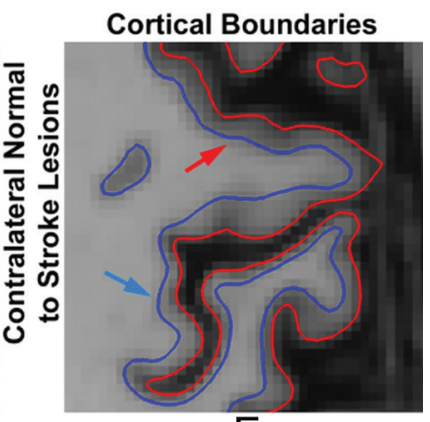

E

FIG 2. A 71-year-old man with acute onset weakness of the left upper extremity presented with an area of acute infarction (blue arrow) along the deep posterior cortex of the right precentral gyrus involving the hand motor region, with $A$, relative hypointensity on MPRAGE, $B$, hyperintensity on FLAIR, and C, restricted diffusion on the ADC map. The patient also has an area of chronic infarction more anteriorly (red arrow), showing $A$, relative hypointensity on MPRAGE, $B$, hyperintensity on FLAIR, and $C, T 2$ shine through on the ADC map. ROIs in the affected areas $(D)$ show higher and lower $C T$, respectively, compared with contralateral analogous brain $(E)$; however, $\mathrm{mGM}$ as a marker of cortical volume was higher in the acute infarct, as expected, but also higher in the chronic infarct. 


\section{Example 1 (concordant)}

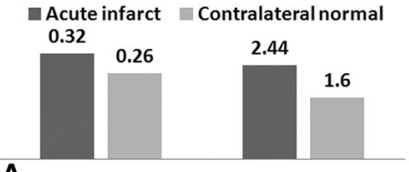

A mGM density
Example 2 (concordant)

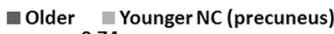

Example 3 (discordant)

@ Chronic infarct $\square$ Contralateral normal 0.58
Example 4 (discordant)

nTBI Matched NC (precuneus)

\begin{tabular}{lllll}
0.52 & 0.58 & 2.12 & 2.07 \\
\hline
\end{tabular}

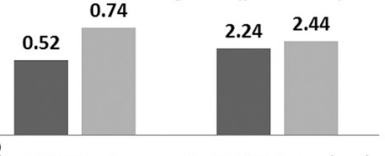

Cortical Thickness $(\mathrm{mm})$

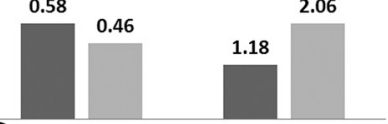

mGM density

Cortical Thickness (mm)

D

mGM density

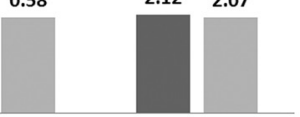

Cortical Thickness (mm)

FIG 3. In vivo examples illustrate concordant results between FSL-VBM and FreeSurfer-CT in the ROls of acute infarct $(A)$ as well as when comparing younger and older healthy control patients $(B)$, and discordant cortical morphometry results in ROls of chronic infarct $(C)$ and in the precuneus $(D)$ in a patient with $\mathrm{mTBI}$ compared with a matched control patient. Of note, prior work reports morphometric changes to the precuneus in aging and traumatic brain injury. The mean values within the ROIs were reported.

VBM and FreeSurfer-CT. Future studies with larger cohorts would be useful to study specific conditions; these results from single patients demonstrate that discordance may be present not only at a group-wise statistical level, but on an individual basis.

\section{CONCLUSIONS}

In summary, we demonstrated the dependence of mGM on T1 signal. Care should be taken in interpreting mGM results as volume change alone. Used in concert, FSL-VBM and FreeSurfer-CT analyses may be complementary.

Disclosures: Yvonne Lui-RELATED: Grant: NIH R01 NS039135-11, NIH R21 NS090349, NIH P41 EB017183. * *Money paid to the institution.

\section{REFERENCES}

1. Good CD, Johnsrude IS, Ashburner J, et al. A voxel-based morphometric study of ageing in $\mathbf{4 6 5}$ normal adult human brains. Neuroimage 2001;14:21-36 CrossRef Medline
2. Dale AM, Fischl B, Sereno MI. Cortical surface-based analysis. I. Segmentation and surface reconstruction. Neuroimage 1999;9:179-94 CrossRef Medline

3. Hutton C, Draganski B, Ashburner J, et al. A comparison between voxel-based cortical thickness and voxel-based morphometry in normal aging. Neuroimage 2009;48:371-80 CrossRef Medline

4. Winkler AM, Kochunov P, Blangero J, et al. Cortical thickness or grey matter volume? The importance of selecting the phenotype for imaging genetics studies. Neuroimage 2010;53:1135-46 CrossRef Medline

5. Smith SM, Brady JM. SUSAN—A new approach to low level image processing. Int J Comput Vis 1997;23:45-78 CrossRef

6. Gaetz W, Roberts TP, Singh KD, et al. Functional and structural correlates of the aging brain: relating visual cortex (V1) gamma band responses to age-related structural change. Hum Brain Mapp 2012; 33:2035-46 CrossRef Medline

7. Zhou Y, Kierans A, Kenul D, et al. Mild traumatic brain injury: longitudinal regional brain volume changes. Radiology 2013;267:880-90 CrossRef Medline 